\title{
SPACE INVOLUTIONS DEFINED BY A WEB OF QUADRICS*
}

\author{
BY \\ VIRGIL SNYDER AND F. R. SHARPE

\section{Properties of the involution}

1. Statement of problem. The following paper is concerned with the study of the transformation between the two spaces $\left(x^{\prime}\right),(x)$ which is defined by the equations

$$
\rho x_{i}^{\prime}=\phi_{i}(x)
$$

$$
(i=1,2,3,4),
$$

where $\phi_{i}(x)=0$ is the equation of a quadric surface. It has been treated synthetically by Reyef but only incidentally in connection with line congruences. In the existing memoirs the involution of the whole of space is not considered except in the special case in which the system of quadrics have six common points; this special case has been extensively studied. $\ddagger$

2. Images of planes, lines, and points. A plane $s_{1}$ in $\left(\dot{x}^{\prime}\right)$ goes into a quadric surface $s_{2}$ in $(x)$, belonging to a definite web. A line $c_{1}^{\prime}$ in $\left(x^{\prime}\right)$ has for image a space quartic $c_{4}$ of genus 1 in $(x)$; the line is the basis of a pencil of planes, and the image quartic is the basis of the pencil of image quadric surfaces. A point $P^{\prime}$ in $\left(x^{\prime}\right)$ has 8 image points in $(x)$; the point $P^{\prime}$ is the vertex of a bundle of planes, and the image points are the eight basis points of the bundle of image quadric surfaces.

A plane $s_{1}$ in $(x)$ goes into a Steiner surface $s_{4}^{\prime}$ in $\left(x^{\prime}\right)$, since by means of the equation of the plane, the coördinates of a point on the image surface are expressible às quadratic functions of three homogeneous parameters. Since the coefficients $a_{i}$ in the equation $\sum a_{i} x_{i}=0$ of a plane do not enter the equation of the image surface linearly, the system of Steiner surfaces in $\left(x^{\prime}\right)$, which appear as images of the whole system of planes of $(x)$, is not linear.

* Presented to the Society, September 4, 1917.

$\dagger \mathrm{T}$. Reye, Ueber die reciproke Verwandtschaft von $F$ Systemen und $\phi^{2}$-Geweben und die quadratischen $F^{2}-$ Systeme achter Stufe, J o u rn al $\mathrm{f} \ddot{\mathrm{u}} \mathrm{d}$ i e r e in e und ange wand te M a th e matik, vol. 82 (1876), pp. 173-206, and Ueber Strahlensysteme zweiter Classe und die Kummersche Fläche vierter Ordnung mit sechzehn Knotenpunkten, ibid., vol. 86 (1879), pp. 84-107.

$\ddagger$ For the literature of this case, see Snyder, An application of the $(1,2)$ quaternary correspondence to the Kummer and Weddle surfaces, these T R A N S A C T I O N S, vol. 12 (1911), pp. 354-366. 
A line $c_{1}$ in $(x)$ goes into a conic $c_{2}^{\prime}$ in $\left(x^{\prime}\right)$, since by means of the equations of $c_{1}$ the coördinates of a point on the image locus can be expressed as quadratic functions of two homogeneous parameters.

A point $P$ in $(x)$ has a single point $P^{\prime}$ in $\left(x^{\prime}\right)$ for image; this appears directly from equation (1).

3. Surfaces of coincidences and of branch points. A point in $\left(x^{\prime}\right)$ which has two coincident images in $(x)$ is called a branch-point. Let $P^{\prime}$ be a branch-point and $P$ the corresponding coincidence. To the bundle of planes through $P^{\prime}$ corresponds a bundle of quadrics having a common tangent line at $P$. Let $P \equiv(0,0,0,1)$, and let $x_{1}=0, x_{2}=0$ be the equations of the common tangent line. We may take the equations of the three linearly independent quadrics through $P$ in the forms $x_{1} x_{4}+f=0, x_{2} x_{4}+\psi=0$, $\left(a x_{1}+b x_{2}\right) x_{4}+\theta=0$, wherein $f, \psi, \theta$ are quadratic forms in $x_{1}, x_{2}, x_{3}$. In this bundle is a cone having its vertex at $P$, hence we have the theorem:

TheOREM I: The surface of coincidences is the locus of the vertices of the cones contained in the web of quadrics.

The equation $\sum \lambda_{i} \phi_{i}=0$ represents a cone when each of its first partial derivatives as to $x_{i}$ vanishes. By eliminating $\lambda_{i}$ from these equations we obtain the equation of the surface of coincidences; it is the jacobian of the web of quadrics. It will be denoted by $K_{4}$. The $c_{4}$ image of a straight line of $\left(x^{\prime}\right)$ meets $K_{4}$ in 16 points, hence:

THEOREM II: The locus of branch points is a surface of order 16 .

It will be denoted by $L_{16}^{\prime}$. The complete image of $L_{16}^{\prime}$ is of order 32; it consists of $K_{4}$ counted twice, and of a residual surface of order 24 , which we denote by $R_{24}$.

A quadric of the web meets $K_{4}$ in a $c_{8}$ of genus 9 , hence a plane section of $L_{16}^{\prime}$ is of genus 9 . A cone of the web meets $K_{4}$ in a $c_{8}$ of genus 8 , having a double point at the vertex of the cone. The image plane meets $L_{16}^{\prime}$ in a curve of genus 8 , having a double point at the image point of the vertex of the cone. It is a tangent plane to $L_{16}^{\prime}$, hence we may say:

THEOREM III: The images of the quadric cones of the web are the tangent planes to $L_{16}^{\prime}$.

We shall now prove the theorem:

Theorem IV: The surface $K_{4}$ contains ten straight lines. We have seen that in the web of quadrics are $\infty^{2}$ cones, the vertices of which lie on $K_{4}$. If a quadric of the web is composite, every point of its line of vertices lies on $K_{4}$; if $(x)$ is any point on the line common to the two component planes, then for $(x)$ every first minor of the discriminant of the quadric must vanish. In the system $\sum \lambda_{i} \phi_{i}=0$ are ten composite quadrics.*

\footnotetext{
* Salmon, Algebra. Lesson 19 in the 4th edition, 1885.
} 
4. Particular lines. Given a point $P^{\prime}$ in $\left(x^{\prime}\right)$. Its images in $(x)$ are $P_{1}, P_{2}, \cdots, P_{8}$. The bundle of planes through $P^{\prime}$ goes into the bundle of quadrics through the eight associated points $P_{i}$. A straight line through $P^{\prime}$ is determined by one other point; a $c_{4}$ through all $P_{i}$ is determined by one other point. If an additional point is chosen on the straight line $P_{i} P_{k}$, the associated $c_{4}$ has three collinear points, hence consists of the line $P_{i} P_{k}$ and a space cubic which meets the line in two points. The image of this composite quartic is a straight line in $\left(x^{\prime}\right)$ which touches $L_{16}^{\prime}$ at the images of the points of intersection of $P_{i} P_{k}$ and its residual cubic. Thus, any line in $(x)$ which joins two associated points has a line for image in $\left(x^{\prime}\right)$. Between these two lines exists a $(1,2)$ correspondence, the double points of which are the points of intersection of $P_{i} P_{k}$ and $K_{4}$, not on the residual cubic. The lines $P_{i} P_{k}$ are all double tangents to $R_{24}$. Through any point $P$ of $(x)$ seven such bitangents can be drawn. The image lines are all bitangents to $L_{16}^{\prime}$. Through any point $P^{\prime}$ of $\left(x^{\prime}\right)$ twenty-eight such bitangents can be drawn, since there are 28 lines $P_{i} P_{k}$ associated with any point $P^{\prime}$.

5. Images of the ten lines on $K_{4}$. It has been seen that the web of quadrics $\sum \lambda_{i} \phi_{i}=0$ contains ten pairs of planes, and that the line of intersection of the planes of each pair lies on $K_{4}$. The image of each line $\gamma_{i}$ is a conic $\gamma_{i}^{\prime}$ on $L_{16}^{\prime}$. Since the pair of planes constitutes a quadric of the web, and the image of a quadric of the web is a plane, it follows that the plane of the conic is the image plane of the composite quadric. Since every point of the line is on $K_{4}$, the plane of the image conic touches $L^{\prime}$ at every point of the conic.

The curve of intersection of a quadric of the web with $K_{4}$ has for image a plane section of $L_{16}^{\prime}$. In the case of a composite quadric, the curve of intersection with $K_{4}$ consists of the line of vertices taken twice and of two elliptic cubic curves. Hence the plane of each singular conic $\gamma_{i}^{\prime}$ meets $L_{16}^{\prime}$ in two elliptic curves, each of order six.

6. Successive images of a plane. A plane $s_{1}$ of $(x)$ goes into a Steiner surface $s_{4}^{\prime}$, having three concurrent double lines $p_{1}^{\prime}, p_{2}^{\prime}, p_{3}^{\prime}$.

The complete image of $s_{4}^{\prime}$ consists of $s_{1}$ and of a residual surface $s_{7}^{\prime}$ of order 7 . The plane $s_{1}$ meets $K_{4}$ in a plane quartic curve through which $s_{7}^{\prime}$ passes; the residual intersection of $s_{1}$ and $s_{7}^{\prime}$ consists of three lines $p_{1}, p_{2}, p_{3}$, images of the double lines of $s_{4}^{\prime}$. The curve $\left(s_{1}, K_{4}\right)$ goes over into a curve of order 8 which is a curve of contact of $s_{4}^{\prime}$ and $L_{16}^{\prime}$. The residual curve of intersection of $s_{4}^{\prime}$ and $L_{16}^{\prime}$ is a $c_{48}^{\prime}$; it is the image of the curve $\left(s_{1}, R_{24}\right)$.

The congruence of lines $P_{i} P_{k}$ in $(x)$ is of order 7 and class 3; it has $R_{24}$ for focal surface.

A general line $c_{1}$ meets $K_{4}$ in 4 points; its image in $\left(x^{\prime}\right)$ is a conic $c_{2}^{\prime}$ which touches $L_{16}^{\prime}$ in the images of the points of intersection. The residual 24 points of intersection of $c_{2}^{\prime}$ and $L_{16}^{\prime}$ are images of the points $\left(c_{1}, R_{24}\right)$. 
A line $P_{i} P_{k}$ meets $K_{4}$ in four points, two of which are on the residual $c_{3}$. The image line is bitangent to $L_{16}^{\prime}$ and meets it in 12 other points. The points of tangency are images of the points $\left(c_{1}, c_{3}\right)$, and they have points of tangency of $c_{1}$ and $R_{24}$ for residual images. Two of the residual points of $c_{1}^{\prime}, L_{16}^{\prime}$ are coincidences on $K_{4}$; the remaining points have each a pair of intersections of $c_{1}$ with $R_{24}$ for images. The same 10 points have for residual images the 10 residual intersections of $c_{3}$ with $K_{4}$.

7. Two planes and their images. Let $p_{1}, p_{2}$ be two planes in $(x)$, and $s_{1}^{\prime}, s_{2}^{\prime}$ their image Steiner surfaces in $\left(x^{\prime}\right)$. The image of the line $\left(p_{1}, p_{2}\right)$ is the conic $p^{\prime}$ common to both Steiner surfaces, which also intersect in a residual $c_{14}^{\prime}$ having 10 points on $p^{\prime}$. To obtain further properties, pass a plane through a double iine $c_{1}^{\prime}$ of $s_{1}^{\prime}$. It meets $s_{1}^{\prime}$ in a conic $c_{2}^{\prime}$ and $s_{2}^{\prime}$ in a quartic $c_{4}^{\prime}$. The line $c_{1}^{\prime}$ meets $s_{2}^{\prime}$ in four points $P_{1}^{\prime}, \cdots, P_{4}^{\prime}$; through $P_{1}^{\prime}$ pass $p^{\prime}$ and $c_{14}^{\prime}$; through each of the others $c_{14}^{\prime}$ passes twice. The curves $c_{2}^{\prime}, c_{4}^{\prime}$ meet in 8 points, through 7 of which $c_{14}^{\prime}$ passes, and the other lies on $p^{\prime}$. The partial image of $c_{1}^{\prime}$ is $c_{1}$ in $p_{1}$. The residual $s_{7}^{\prime}$, image of $s_{2}^{\prime}$, meets $p_{1}$ in a curve of order 7 , having one point on $\left(p_{1}, p_{2}\right)$. The other six are three pairs of images of $P_{2}^{\prime}, P_{3}^{\prime}, P_{4}^{\prime}$. The plane through $c_{1}^{\prime}$ goes into a quadric of the web through $c_{1}$. The plane $p_{1}$ cuts the quadric in $c_{1}$ and another line $\vec{c}_{1}$.

The plane $p_{2}$ meets the quadric in a conic $c_{2}$ which has a point on $c_{1}$ and one on $\bar{c}_{1}$. The image of $\bar{c}_{1}$ is the conic $c_{2}^{\prime}$, and that of $c_{2}$ is $c_{4}^{\prime}$. Since $\left(p_{1}, p_{2}\right)$, $\bar{c}_{1}$, and $c_{2}$ have a point in common, it follows that $p^{\prime}, c_{2}^{\prime}, c_{4}^{\prime}$ have a point in common. The curve $c_{14}^{\prime}$ does not pass through this point. Since $c_{14}^{\prime}$ has three double points and one simple intersection on each double line of $s_{1}^{\prime}, s_{2}^{\prime}$, its genus is 6 .

8. Double curve and cuspidal curve on $L_{16}^{\prime}$. It has been seen that the locus of points in $\left(x^{\prime}\right)$ which have two coincident images in $(x)$ is $L_{16}^{\prime}$, and that the locus of the coincidences is $K_{4}$. On $L_{16}^{\prime}$ is a cuspidal curve, each point of which has at least three coincident images and a double curve, each point of which has a pair of coincident images. The points of intersection of these two curves are either points to which correspond four coincident images, or a coincidence of three image points at one point and a simultaneous coincidence of two at another. The orders of these curves are the number of cusps and double points in a general plane section of $L_{16}^{\prime}$.

Since the order and genus of a general plane section are known, all the characteristic numbers can be determined when one more is known. The bitangents of the plane section are the lines whose images in $(x)$ are composite quartic curves. Of the bitangents, 10 are the lines in which the plane of section meets the ten singular planes. The image of the plane of section is a quadric of the web. If a line in $\left(x^{\prime}\right)$ has for image in $(x)$ a line and a residual cubic, the image line is a generator of the quadric and joins a pair of associated points $P_{i}, P_{k}$. 
Such a line is a component of the basis curve of a pencil of quadrics contained in the web. Let $x_{1} x_{3}-x_{2} x_{4}=0$ be the equation of a quadric. A generator of one system has equations $x_{1}=\lambda x_{2} ; \lambda x_{3}=x_{4}$. Any quadric of the web has an equation of the form $\sum a_{i} \phi_{i}=0$. Replace $x_{1}$ by $\lambda x_{2}, x_{4}$ by $\lambda x_{3}$, equate the coefficients of $x_{2}^{2}, x_{2} x_{3}, x_{3}^{2}$ to zero and eliminate the $a_{i}$. The result is a sextic in $\lambda$, hence 6 generators of the $\lambda$-system join associated points. The same quadric contains 6 generators of the other stysem which join associated points.

Hence the total number of bitangents is 22 . From Plücker's equations we now obtain $\delta=60, \kappa=36$, from which we may state the theorem:

Theorem. The surface $L_{16}^{\prime}$ has a double curve of order 60 and a cuspidal curve of order 36 .

9. Contact and intersection curves on $K_{4}$. When the point $P^{\prime}$ describes $c_{36}^{\prime}$ on $L_{16}^{\prime}$, the triple image $P$ is on $K_{4}$, and the remaining 5 images are on $R_{24}$. But $P$ is also on $R_{24}$, and since a definite direction is associated with every point of $K_{4}$ (the limit of the direction $P_{i} P_{k}$ as $P_{i} \doteq P_{k}$ ) it follows that $K_{4}$ and $R_{24}$ touch each other along the curve described by $P$. This curve is in $(1,1)$ correspondence with $c_{36}^{\prime}$, and is of order 18 . When $P^{\prime}$ is a point on $c_{60}^{\prime}$ on $L_{16}^{\prime}$, two of its images coincide at $P_{1}$, two more at $P_{2}$, and there are four other images on $R_{24}$. $\quad P_{1}$ is on $K_{4}$, hence $P_{2}$ is on $R_{24}$; but $P_{2}$ is on $K_{4}$, hence $P_{1}$ is on $R_{24}$. Hence $K_{4}, R_{24}$ intersect along the curve described by $P_{1}$ and $P_{2}$ as $P^{\prime}$ describes $c_{60}^{\prime}$. The locus of $P_{1}\left(\right.$ and $P_{2}$ ) is of order 60; its image in $\left(x^{\prime}\right)$ is $c_{60}^{\prime}$ counted twice.

10. Special properties of $\gamma_{i}, \gamma_{i}^{\prime}$. The image of any straight line $\gamma$ on $K_{4}$ is a singular conic $\gamma^{\prime}$ on $L_{16}^{\prime}$ (No. 5). The complete image of $\gamma^{\prime}$ consists of $\gamma$ counted twice and of a residual cubic $g_{3}$ in each component plane $\pi_{1}, \pi_{2}$ of the composite quadric of the web belonging to $\gamma$. These two cubics both lie on $R_{24}$, but not on $K_{4}$. Each plane touches $R_{24}$ along $g_{3}$. Each plane $\pi_{i}$ meets $K_{4}$ in a cubic $c_{3}$, passing through the three points $\left(\gamma, g_{3}\right)$, and meeting $g_{3}$ in six others. The image of each $c_{3}$ is a $c_{6}^{\prime}$ in the plane $\pi^{\prime}$ of $\gamma^{\prime}$. Together they constitute the residual intersection of $\pi^{\prime}$ with $L_{16}^{\prime}$. Each $c_{6}^{\prime}$ touches $\gamma^{\prime}$ in three points, images of the points $\left(\gamma, c_{3}\right)$, and cuts it in six points, images of the intersections $\left(g_{3}, c_{3}\right)$. The complete image of $c_{6}^{\prime}$ is $c_{3}$ counted twice, a residual $c_{6}$ in $\pi_{1}$, and a $c_{12}$ in $\pi_{2}$. Each plane $\pi$ is tangent to the contact curve $c_{18}$ in the three points $\left(\gamma, c_{3}\right)$. The remaining 9 points of intersection of $\pi$ with $c_{18}$ (not on $\gamma$ ) are on $c_{3}$, hence $c_{6}^{\prime}$ has 9 cusps. At each of the three contacts of $c_{6}^{\prime}$ with $\gamma^{\prime}$ the plane $\pi^{\prime}$ meets the cuspidal curve $c_{36}^{\prime}$ in three points. At each intersection of $c_{6}^{\prime}$ with $\gamma^{\prime}$ the plane $\pi^{\prime}$ touches the double curve $c_{60}^{\prime}$; the residual intersections of $c_{60}^{\prime}$ with $\pi^{\prime}$ are at the 36 points common to the two sextics.

11. Complete images of cuspidal and double curves. The complete image 
of $c_{36}^{\prime}$ is $c_{18}$ counted three times and a residual $c_{90}$ which is a cuspidal curve on $R_{24}$. The image of $c_{60}^{\prime}$ is $c_{60}$ counted twice and a residual $c_{120}$ which is a double curve on $R_{24}$. The curve $c_{90}$ meets $K_{4}$ in 360 points. Let $t$ of these points $T$ be on $c_{18}$ and $c_{60}$, that is, points at which four images of a point in $\left(x^{\prime}\right)$ coincide; let there be $m$ points $M$ on $c_{60}$ such that for each $M$ the corresponding point in $\left(x^{\prime}\right)$ has two images at $M$ and three at an intersection of $c_{18}$ and $c_{60}$. At the points $T$ the curve $c_{90}$ has contact with $K_{4}$, hence

$$
2 t+m=360 \text {. }
$$

The curve $c_{120}$ meets $K_{4}$ in 480 points. Let there be $p$ points in $\left(x^{\prime}\right)$ whose images in $(x)$ consist of three coincidences at $P_{1}, P_{2}, P_{3}$, and of two residual points. We have the relation

$$
3 p+m=480 .
$$

If we apply the Zeuthen formula for the number of coincidences in a multiple correspondence to the $(2,1)$ correspondence between $c_{60}, c_{60}^{\prime}$ and the $(4,1)$ between $c_{120}, c_{60}^{\prime}$ we obtain the relation

Hence

$$
p=t .
$$

$$
p=t=m=120 .
$$

The curves $c_{18}, c_{60}$ touch in $t$ points $T$, and intersect in the $m$ points $M$. Hence they have $2 t+m=360$ common points.

12. Web with one basis point. Let $A=(0,0,0,1)$ be on all the quadrics of the web. The equations of transformation may be expressed in the form

$$
\begin{aligned}
& \rho x_{i}^{\prime}=x_{4} x_{i}+\phi_{i} \\
& (i=1,2,3), \\
& \rho x_{4}^{\prime}=\phi_{4},
\end{aligned}
$$

wherein $\phi_{i}$ are homogeneous and quadratic in $x_{1}, x_{2}, x_{3}$. The point $A$ is a fundamental point; its image is the plane $x_{4}^{\prime}=0$. Not all the images of a point in $x_{4}^{\prime}=0$ are at $A$; the residual locus is the cone $\phi_{4}=0$ having $A$ for vertex.

Every line through $A$ joins two associated points, hence each has a line in $\left(x^{\prime}\right)$ for image. The image of the line $x_{i}=\lambda_{i} r(i=1,2,3)$ is the line

$$
x_{i}^{\prime}=x_{4} \lambda_{i}+\phi_{i}(\lambda) r, \quad x_{4}^{\prime}=\phi_{4}(\lambda) r .
$$

The points in which these lines in $\left(x^{\prime}\right)$ meet the singular plane $x_{4}^{\prime}=0$ are projective with the lines of the bundle at $A$. To the directions of the generators of the cone $\phi_{4}=0$ correspond the points of a singular conic $\phi_{4}^{\prime}=0$ in $x_{4}^{\prime}=0$. The cone $\phi_{4}=0$ is the tangent cone to $K_{4}$ at $A$, which is a double point on the surface. 
Two general quadrics of the web intersect in a quartic curve which meets $K_{4}$ in 14 points apart from $A$. Hence $L^{\prime}$ is of order 14 .

A quadric of the web meets $K_{4}$ in a curve of order 8 and genus 8 , hence a plane section of $L_{14}^{\prime}$ is of genus 8 . The plane $x_{4}^{\prime}=0$ touches $L_{14}^{\prime}$ along the conic $\phi_{4}^{\prime}=0, x_{4}^{\prime}=0$. The surface $K_{4}$ has an equation of the form

$$
x_{4}^{2} \phi_{4}+x_{4} \psi+f=0 .
$$

Each generator of $\phi_{4}=0$ meets $K_{4}$ in one point apart from $A$, on the surface $x_{4} \psi+f=0$. The curve of intersection is of order 8 and has a six-fold point at $A$. The image is the curve

$$
x_{i}^{\prime}=\phi_{i} \psi-f x_{i}, \quad i=1,2,3, \quad x_{i}^{\prime}=0,
$$

in which the parameters $x_{1}, x_{2}, x_{3}$ are connected by the relation $\phi_{4}=0$. This curve is rational and of order 10; it is the residual intersection of $x_{4}^{\prime}=0$ with $L_{14}^{\prime}$. The images of the lines of $\phi_{4}=0$ are tangents to this curve $c_{10}^{\prime}$.

Since $L^{\prime}$ is of order 14 , it follows that the order of $R$ is 20 . A straight line meets $L_{14}^{\prime}$ in 14 points; its image $c_{4}$ meets $R_{20}$ in 70 points not at $A$. Since $c_{4}$ has a simple point at $A$, it follows that $R_{20}$ has a 10 -fold point at $A$.

A general line $l$ through $A$ meets $K_{4}$ in two residual points $P_{1}, P_{2}$. Its image is a line $l^{\prime}$ tangent to $L_{14}^{\prime}$ at $P_{1}^{\prime}, P_{2}^{\prime}$ and meeting $L_{14}^{\prime}$ in ten other points, images of the residual intersections of $l$ with $R_{20}$.

Any point $P^{\prime}$ in $\left(x^{\prime}\right)$ has 8 images in $(x)$, one of which is $A$; through $A$ can be drawn seven lines of the bundle to the other associated points, hence the image congruence in $\left(x^{\prime}\right)$ is of order 7 . Since any quadric of the web contains two lines through $A$, the image congruence is of class 2 . Since the points of $x_{4}^{\prime}=0$ are projective with the lines through $A$, it follows that no two lines not in $x_{4}^{\prime}=0$ can meet in a point of $x_{4}^{\prime}=0$. If $P^{\prime}$ is chosen in $x_{4}^{\prime}=0$, it follows that of the seven lines of the congruence through $P^{\prime}$, six must be in $x_{4}^{\prime}=0$, and be tangent to $c_{10}^{\prime}$. Hence $c_{10}^{\prime}$ is of class 6 ; it must therefore have 24 double points and 12 cusps.

The image of $l^{\prime}$ is $l$ and a residual $c_{3}$ meeting $K_{2}$ (the cone $\phi_{4}=0$ ) in 6 points, but not passing through $A$. The six generators of $K_{2}$ through $\left(c_{3}, K_{2}\right)$ have for images the 6 tangents to $c_{10}^{\prime}$ through $P^{\prime}$. These 6 points and the direction of $l$ at $A$ are the 7 images of $P^{\prime}$, apart from $A$ itself.

The lines of $A$, projective with the points of $c_{10}^{\prime}$, are the generators of the tangent cone $K_{10}$ to $R_{20}$ at $A$. This cone has 24 double generators and 12 cuspidal generators.

A plane section of $L_{14}^{\prime}$ has 23 bitangents, those accounted for in the general case and the section with $x_{4}^{\prime}=0$.

The cuspidal curve of $L_{14}^{\prime}$ is therefore of order 30 , and the double curve of order 40. The contact curve of $R_{20}$ and $K_{4}$ is still of order 18 , and has a 
six-fold point at $A$. The curve of intersection is of order 44 and has an 8-fold point at $A$. The two tangent cones $K_{2}, K_{10}$ touch each other along the six tangents to the contact curve, and intersect in the eight tangents to the curve of intersection.

The curve $c_{10}$ and the conic $\phi_{4}^{\prime}=0$ touch at the six image points in $x_{4}^{\prime}=0$, and intersect at the eight image points. The cuspidal curve $c_{30}^{\prime}$ has second order contact with $\phi_{4}^{\prime}=0$ at the six points, while the double curve $c_{40}^{\prime}$ has simple contact with $\phi_{4}^{\prime}=0$ at the eight points.

The complete image of $c_{30}^{\prime}$ is the contact curve $c_{18}$ counted three times, and a residual cuspidal curve $c_{66}$ on $R_{20}$, having a 12 -fold point at $A$; the tangents at $A$ being the images of the cusps of $c_{10}^{\prime}$. The complete image of $c_{40}$ is the curve of intersection $c_{44}$ counted twice, and a residual double curve $c_{72}$ on $R_{20}$, having a 24 -fold point at $A$, the tangents being the images of the double points of $c_{10}^{\prime}$.

Hence we have

$$
\begin{aligned}
& 2 t+m=264-24=240, \\
& 3 p+m=288-48=240,
\end{aligned}
$$

and from Zeuthen's formula

$$
3 p=2 t \text {. }
$$

The complete image of $c_{10}^{\prime}$ is $c_{8}$ counted twice and a residual $c_{24}$ having an 8 -fold point at $A$. The complete image of $\phi_{4}^{\prime}=0, x_{4}^{\prime}=0$ is $A$ and a curve of order 8 having a six-fold point at $A$. The surfaces $R_{20}, \phi_{4}=0$ touch along this curve and intersect in $c_{24}$.

13. Curves on composite quadrics. Given a line $\gamma$ on $K_{4}$, let $\pi_{1}$ pass through $A$. The image of $\gamma$ is $\gamma^{\prime}$ in $\pi^{\prime}$. Of the eight images in $(x)$ of a point on $\pi^{\prime}$, four are in $\pi_{2}$, and four in $\pi_{1}$, of which one is fixed at $A$. The complete image of $\gamma^{\prime}$ is $\gamma$ counted twice, a nodal cubic $g_{1}$ in $\pi_{1}$, with node at $A$, and a cubic $g_{2}$ in $\pi_{2}$. Both planes touch $R_{20}$ along these cubics. The plane $\pi_{1}$ meets $K_{4}$ in a cubic $c_{3,1}$ having a double point at $A$. Of the nine points $\left(g_{1}, c_{3,1}\right)$, three are on $\gamma$, four are at $A$, and two others. The image of $c_{3,1}$ is $c_{4}^{\prime}$ and of $c_{3,2}$ is $c_{6}^{\prime}$ in $\pi^{\prime}$; together they constitute the residual section of $\pi^{\prime}$ with $L_{14}^{\prime}$. The curve $c_{4}^{\prime}$ touches $\gamma^{\prime}$ in three points, images of $\left(\gamma, c_{3,1}\right)$, and cuts it in two points, images of $\left(g_{1}, c_{3,1}\right)$ not at $A$ nor on $\gamma$. The complete image of $c_{6}^{\prime}$ is $c_{3,2}$ counted twice, a residual $c_{6}$ in $\pi_{2}$, and a $c_{12}$ in $\pi_{1}$, with a six -fold point at $A$. The complete image of $c_{4}^{\prime}$ is $c_{3,1}$ counted twice, a conic in $\pi_{1}$ not passing through $A$, and a $c_{8}$ in $\pi_{2}$.

The plane $\pi_{1}$ meets the contact curve $c_{18}$ in 9 points on $\gamma$, six points at $A$, and three other points on $c_{3,1}$. Hence $c_{4}^{\prime}$ has three cusps.

The plane $\pi^{\prime}$ meets the nodal curve $c_{40}^{\prime}$ in the six points of intersection $\left(c_{6}^{\prime}, \gamma^{\prime}\right)$ each counted twice, the two points of intersection $\left(c_{4}^{\prime}, \gamma^{\prime}\right)$ each counted twice, and in the 24 intersections $\left(c_{4}^{\prime}, c_{6}^{\prime}\right)$. 
14. Web with two basis points. Let $A \equiv(0,0,0,1), B \equiv(0,0,1,0)$ be the two basis points. The equations of transformation may be expressed in the form

$$
\begin{aligned}
& x_{1}^{\prime}=x_{4} x_{3}+a_{1} x_{3} x_{1}+\phi_{1}\left(x_{1}, x_{2}\right), \\
& x_{2}^{\prime}=x_{4} x_{2}+a_{2} x_{3} x_{1}+\phi_{2}\left(x_{1}, x_{2}\right), \\
& x_{3}^{\prime}=x_{4} x_{1}+\phi_{3}\left(x_{1}, x_{2}\right), \\
& x_{4}^{\prime}=\quad a_{4} x_{3} x_{2}+\phi_{4}\left(x_{1}, x_{2}\right) .
\end{aligned}
$$

The image of the point $A$ is the plane $x_{4}^{\prime}=0$, and of the point $B$ is the plane $x_{3}^{\prime}=0$. Any point of the line $A B$ has for image the single point $(A B)^{\prime} \equiv(1,0,0,0)$. The complete image in $(x)$ of $(A B)^{\prime}$ is the line $A B$ and four points not on the line. The image of the line $x_{3}^{\prime}=0, x_{4}^{\prime}=0$ consists of the line $A B$ and the cubic curve $x_{4} x_{1}+\phi_{3}=0, a_{4} x_{3} x_{2}+\phi_{4}=0$ which meets $A B$ in two points. The four residual points, images of $(A B)^{\prime}$, are the intersections of the cubic and the planes

$$
a_{4} x_{2}^{2} \phi_{3}+a_{2} x_{1}^{2} \phi_{4}-a_{4} x_{1} x_{2} \phi_{2}=0 \text { through } A B .
$$

The points $A, B$ are both double points on $K_{4}$; the cone $a_{3} x_{3} x_{2}+\phi_{4}=0$ is tangent cone to $K_{4}$ at $A$, and $x_{4} x_{1}+\phi_{3}=0$ is tangent cone to $K_{4}$ at $B$. The line $A B$ lies entirely on $K_{4}$.

The surface $L^{\prime}$ is of order 12; the planes $x_{3}^{\prime}=0, x_{4}^{\prime}=0$ are both singular, touching it along conics. The point $(A B)^{\prime}$ lies on both conics and is a double point on $L^{\prime}$. The residual surface $R$ is of order 16; it contains the residual cubic, image of $x_{3}^{\prime}=0, x_{4}^{\prime}=0$.

The image of a line $l$ through $A$ is a line $l^{\prime}$ meeting $x_{4}^{\prime}=0$ at $Q^{\prime}$ and $x_{3}^{\prime}=0$ at $T^{\prime}$. The point $Q^{\prime}$ is the image of the direction of $l$ at $A ; T^{\prime}$ is the image of the direction of the cubic curve, residual to $l$, at $B$. The line $l^{\prime}$ is bitangent to $L_{12}^{\prime}$ and meets it in 8 other points. Hence $l$ meets $R_{16}$ in 8 points apart from $A$, so that $A$ is an 8-fold point on $R_{16}$. Similarly, $B$ is 8 -fold on $R_{16}$. The line $A B$ does not lie on $R_{16}$.

The residual cubic of $l$ meets the cone $a_{4} x_{3} x_{2}+\phi_{4}=0$ at $B$ and at five other points. The five generators of the cone which pass through these points have for images five tangents to the curve $c_{8}^{\prime}$, the residual section of $L_{12}^{\prime}$ by $x_{4}^{\prime}=0$, apart from the conic of contact. The curve $c_{8}^{\prime}$ is of class 5 and is rational, hence it has 12 double points and 9 cusps. The tangent cone $K_{8}$ to $R_{16}$ at $A$ has therefore 12 double generators and 9 cuspidal generators.

A general quadric of the web meets $K_{4}$ in a curve of genus 7 , hence a plane section of $L_{12}^{\prime}$ is of genus 7; since it has 24 bitangents, we conclude that the double curve of $L_{12}^{\prime}$ is of order 24 , and the cuspidal curve is of order 24 . The curve $c_{8}^{\prime}$ touches the singular conic of its own plane in 5 points, and intersects 
it in 6 . The contact curve of $R_{16}, K_{4}$ is of order 17 , and has a 5 -fold point at $A$ and at $B$. The curve of intersection is of order 30 and has a 6 -fold point at $A$ and at $B$. The tangent cones to $K_{4}$ and $R_{16}$ at $A$ touch along the generators which are images of the points of contact of $c_{8}^{\prime}$ and the singular conic in $x_{4}^{\prime}=0$; they intersect along the generators which are images of the six points of intersection. The cuspidal curve on $R_{16}$ is of order 45 , with $A$ and $B$ as 9-fold points; the double curve is of order 36 , having $A, B$ each 12 -fold.

We now have the relations

$$
\begin{aligned}
2 t+m & =180-36=144, \\
3 p+m & =144-48=96, \\
3 p & =t,
\end{aligned}
$$

hence $t=m=48, p=16$.

The image of $c_{8}^{\prime}$ is the $c_{7}$, intersection of $K_{4}$ and the tangent cone at $A$, counted twice, and a $c_{18}$ having $A, B$ each 6 -fold. The image of the conic in $x_{4}^{\prime}=0$ is $A B$, and another $c_{7}$ with $A 5$-fold, $B$ simple. The curve $c_{18}$ and this $c_{7}$ counted twice is the complete intersection of $R_{16}$ and $a_{4} x_{3} x_{2}+\phi_{4}=0$.

15. Two basis points; composite quadrics. The lines $\gamma$ are now of two different types. In the first type $\gamma_{1}$ one plane $\pi_{1}$ contains both $A$ and $B$. In the second type $\gamma_{2}$ one plane $\pi$ contains $A$, the other $B$.

The image of $\gamma_{1}$ is $\gamma_{1}^{\prime}$ in $\pi_{1}^{\prime}$. The images of a point in $\pi_{1}^{\prime}$ lie four in $\pi_{2}$, and two in $\pi_{1}$, besides $A$ and $B$. The complete image of $\gamma_{1}^{\prime}$ is $\gamma_{1}$ counted twice, the line $A B$, a conic $g_{1}$ through $A$ and $B$, and a cubic $g_{2}$ in $\pi_{2}$. The plane $\pi_{1}$ meets $K_{4}$ in $\gamma_{1}, A B$, and a conic $c_{2}$ through $A$ and $B$. The conics $g_{1}, c_{2}$ intersect on $\gamma_{1}$. The image of $c_{2}$ is a conic $c_{2}^{\prime}$ having contact with $\gamma_{1}^{\prime}$ at the images of $\left(g_{1}, c_{2}\right)$. The plane $\pi_{2}$ meets $K_{4}$ in a cubic $c_{3}$ whose image in $\pi_{1}^{\prime}$ is $c_{6}^{\prime}$, which touches $\gamma_{1}^{\prime}$ in three points and intersects it in six.

The complete image of $c_{2}^{\prime}$ consists of $c_{2}$ counted twice, no other locus in $\pi_{1}$, and a $c_{4}$ in $\pi_{2}$. The complete image of $c_{6}^{\prime}$ is $c_{3}$ counted twice, a $c_{6}$ in $\pi_{2}$, and a $c_{12}$ in $\pi_{1}$ having a six-fold point at $A$ and at $B$. The plane $\pi_{1}$ meets the contact curve $c_{17}$ in 7 points on $\gamma, 5$ at $A$, and 5 at $B$. The plane $\pi_{1}^{\prime}$ meets the cuspidal curve $c_{24}^{\prime}$ in the two points of contact $\left(\gamma_{1}^{\prime}, c_{2}^{\prime}\right)$ and the three points of contact $\left(\gamma_{1}^{\prime}, c_{6}^{\prime}\right)$, each counted three times, and in the nine cusps of $c_{6}^{\prime}$. The nodal curve $c_{24}^{\prime}$ meets $\pi_{1}^{\prime}$ in the six intersections $\left(\gamma_{1}^{\prime}, c_{6}^{\prime}\right)$ each counted twice, and in the twelve intersections $\left(c_{2}^{\prime}, c_{6}^{\prime}\right)$. The plane $\pi_{2}$ meets the contact curve $c_{17}$ in 8 points on $\gamma_{1}$ and nine points on $c_{3}$.

In case of a line $\gamma_{2}$, the two component planes enter symmetrically. Of the variable images of a point in $\pi_{2}^{\prime}$, three are in each plane. The complete image of $\gamma_{2}^{\prime}$ consists of $\gamma_{2}$ counted twice, and a nodal cubic $g$ in each plane $\pi$. 
The plane $\pi$ meets $K_{4}$ in a nodal cubic $c_{3}$, meeting $g$ in $\left(\gamma_{2}, c_{3}\right)$ and in two other points not at the double point. The image of $c_{3}$ is $c_{4}^{\prime}$, which touches $\gamma_{2}^{\prime}$ in three points, images of $\left(\gamma_{2}, c_{3}\right)$, and intersects it in two points, images of $\left(g, c_{3}\right)$. The complete image of $c_{4}^{\prime}$ is $c_{3}$ counted twice, a $c_{7}$ in $\pi_{2}$ with a double point at $B$, and a $c_{3}$ in $\pi_{1}$ with a double point at $A$. The contact curve $c_{17}$ meets $\pi$ in 9 points on $\gamma_{2}, 5$ points at $A$, and in three points on $c_{3}$. Hence $c_{\mathbf{1}}^{\prime}$ has 3 cusps.

16. Three basis points. Let $A, B, C$ be three points common to all the quadrics of the web. Then $A, B, C$ are all double points on $K_{4}$, and the lines $A B, B C, C A$ lie on the surface, besides the lines $\gamma_{i}$. These three lines go into double points $C^{\prime}, A^{\prime}, B^{\prime}$ on $L^{\prime}$, and the vertices go into conics of contact.

One line $\gamma_{1}$ lies in the plane $A B C$, and one component plane of the composite quadric to which it belongs is the plane $A B C$. The image of this composite quadric is the piane $A^{\prime} B^{\prime} C^{\prime}$.

The bundle $A, B, C$ have congruences of bitangents of $L^{\prime}$ for images, each of order 5 and class 2 .

$L^{\prime}$ is now of order 10 , and any plane section is of genus 6 , since it is the image of a $(4,4)$ curve on a quadric, but having three double points. The cuspidal curve on $L_{10}^{\prime}$ is of order 18; the double curve. is of order 12 .

The surface $R$ is of order 12 , and has $A, B, C$ for six-fold points. On $L_{10}^{\prime}$ the cuspidal curve is of order 18 , and the double curve of order 12 . The contact curve of $K_{4}, R_{12}$ is of order 15 , and has $3 P_{4}$. The curve of intersection is of order 18 and has $3 P_{4}$. The cuspidal curve on $R_{12}$ is of order 27 and has $3 P_{6}$. The double curve on $R_{12}$ is of order 12 and has $3 P_{4}$. The tangent cones $A, B, C$ have 4 tangents with 4 point contact with $K_{4}$. In $\alpha^{\prime}$, the residual curve to the singular conic is of order 6 , class 4 , and genus 0 ; it has 6 cusps and 4 double points.

This curve meets the conic in 4 contacts, each counting for 3 cusps, and 4 intersections, each counting as 2 double points.

We now have

Hence

$$
\begin{aligned}
2 t+m & =108-36=72, \\
m & =48-24=24, \\
p & =0 .
\end{aligned}
$$

$$
t=m=24 \text {. }
$$

The lines $\gamma$ are of two kinds; $\gamma_{1}$ lies in the plane $A B C$, and the other component plane passes through no basis point. The image $\gamma_{1}^{\prime}$ passes through the three double points of $L_{10}^{\prime}$. The complete image of $\gamma_{1}^{\prime}$ consists of $\gamma_{1}$ counted twice, the three lines $A B, B C, C A$, and a cubic $g_{3}$ in $\pi_{2}$. 
The plane $A B C$ of $K_{4}$ has no residual curve; the plane $\pi_{1}$ meets $K_{4}$ in a cubic $c_{3}$ whose image in $\pi_{1}^{\prime}$ is a $c_{6}^{\prime}$. The contact curve $c_{15}$ has 9 points on $c_{3}$, hence $c_{6}^{\prime}$ has 9 cusps. It touches $\gamma_{1}^{\prime}$ in three points and meets it in six.

The composite quadrics through the remaining lines $\gamma_{2}$ consist of a plane $\pi_{1}$ through two basis points, and a plane $\pi_{2}$ through the other, hence $\gamma_{2}$ meets but one line as $A B$. The image conic $\gamma_{2}^{\prime}$ passes through one double point. The complete image in $(x)$ of $\gamma_{2}^{\prime}$ consists of $\gamma_{2}$ counted twice, the line $A B$, a conic $g_{2}$ in $\pi_{1}$ through $A$ and $B$, and a cubic $g_{3}$ in $\pi_{2}$, having a double point at $C$. The plane $\pi_{1}$ meets $K_{4}$ in a conic $c_{2}$ through $A$ and $B$, and meeting $g_{2}$ on $\gamma_{2}$; the plane $\pi_{2}$ meets $K_{4}$ in a cubic $c_{3}$ having a double point at $C$. The curves $\left(g_{3}, c_{2}\right)$ meet in two points apart from $\gamma_{2}$ and $C$. The image of $c_{2}$ is $c_{2}^{\prime}$ having double contact with $\gamma_{2}^{\prime}$; the image of $c_{3}$ is $c_{4}^{\prime}$ touching $\gamma_{2}^{\prime}$ in 3 points.

The plane $\pi_{1}$ meets the contact curve $c_{15}$ in 7 points on $\gamma_{2}$ and 8 points at $A$ and $B$; the plane $\pi_{2}$ meets it in 8 points on $\gamma_{2}, 4$ points at $C$, and 3 points on $c_{3}$. Hence $c_{4}^{\prime}$ has 3 cusps.

17. Four basis points. Let $A, B, C, D$, the vertices of a proper tetrahedron, be fixed basis points. $K_{4}$ now has four double points and six additional lines. The surface $L^{\prime}$ is of order 8 , has 14 singular planes, and 6 nodes. A plane section is of genus 5 . The cuspidal curve is of order 12 , and the double curve is of order 4 .

The bundles of lines through $A, B, C, D$ have for images congruences of order 4 , class 2 , having $L_{8}^{\prime}$ for complete focal surface.

The surface $R$ is of order 8 , and has a 4 -fold point at each basis point. The contact curve is of order 12 and has 4 three-fold points; the curve of intersection is of order 8 , and has double points at the basis points.

We now have

$$
p=0, \quad m=0, \quad t=12 \text {. }
$$

The lines $\gamma$ are again of two kinds; in one case, $\pi_{1}$ contains three points, $\pi_{2}$ one; in the other, each contains two. The image plane of the former contains a $c_{4}^{\prime}$ with three cusps; in the latter, we have two conics, each having double contact with $\gamma^{\prime} . \quad R_{8}$ has a cuspidal curve of order 12 , having a triple point at each basis point. It has no double curve.

18. Five basis points. The surface $K_{4}$ now has five double points and ten additional lines. The surface $L^{\prime}$ is of order 6 , has fifteen singular planes, and ten double points. It has a cuspidal curve of order 6 , and no double curve. The surface $R$ is of order 4 and has the basis points for nodes. It has no cuspidal or double curve. The curve of contact is of order 8 and has double points at the basis points. There is no curve of intersection. $K_{4}, R_{4}, L_{6}^{\prime}$ are all in $(1,1)$ correspondence.

In this case

$$
p=m=t=0
$$


The lines $\gamma$ are all of the same kind; one plane passes through three basis points, the other through two. In every singular plane the residual section is a conic having double contact with the singular conic.

The images of the 5 bundles are congruences of order 3 , class 2 , having $L_{6}^{\prime}$ for complete focal surface.

19. Web with a basis line. Most of the properties of this and other special cases have already been developed.*

I.et $d$ be the common basis line. It is a three-fold line on $K_{4}$, which is now a ruled surface. Every coincidence not on $d$ belongs to a composite quadric. The images of the points of $d$ are generators of a quadric $F_{2}^{\prime}$ in $\left(x^{\prime}\right)$. $L^{\prime}$ is a developable surface of order 6 ; the cuspidal curve is of order 6 , and the double curve of order 4 . The image of $L_{6}^{\prime}$ consists of $K_{4}$ taken twice and a developable quartic having a cuspidal cubic and no double curve. The image of the cuspidal curve $c_{b}^{\prime}$ consists of a curve of contact $c_{5}^{\prime}$ of order 5 counted three times, meeting $d$ in four points, and the cuspidal curve of $R_{4}$. The image of the double curve $c_{4}^{\prime}$ is the curve of intersection of $K_{4}, R_{4}$. It is of order 6 and meets $d$ in the points $\left(d, c_{5}^{\prime}\right)$. The configurations in the cases having additional basis elements can now be obtained readily. Similarly for other special webs. $\dagger$

\section{Transformations of $K_{4}$}

20. Systems of curves on $K_{4}$. The surface $K_{4}$ is a particular case of those having an equation of the form $\left|a_{1} b_{2} c_{3} d_{4}\right|=0$, in which the elements are general linear functions of $(x)$. The general surface contains two triply infinite systems of sextics of genus $3, C_{6}$ and $\bar{C}_{6}$, each cut from the surface by the cubics through a curve of the other. The system of plane sections $\left|C_{4}\right|$ and either system of sextics constitute a minimum base on the surface. In the case of $K_{4}$ it is necessary to eniarge the base on account of the lines $\gamma_{i}$. We shall now prove the following theorem.

Theonem. The basis number of $K_{4}$ is 11 . The systems $\left|C_{4}\right|,\left|C_{6}\right|$ and nine lines $\gamma_{i}$ constitute a minimum basc.

Consider the transformation defined by $r_{i}^{\prime}=D_{i}(x), D_{i}$ being the cofactor of $d_{i}$ in the determinant $\left|a_{1} b_{2} c_{3} d_{4}\right|$. It is involutorial and under it

$$
C_{4} \sim C_{6}, \quad C_{6} \sim C_{4} .
$$

The image of any line $\gamma_{i}$ is a space cubie $\left(\gamma_{i}\right)$. Since $\left[C_{4},\left(\gamma_{i}\right)\right]=3$, hence $\gamma_{i}$ is a trisecant of every curve of the system $C_{6}$. Any cubic $F_{3}$ of the system

* See Encyklopädie der mathematischen Wissenschaften, III, C, 2, no. 143 for the literature.

$\dagger$ In particular see Encyklopädic, l. c., no. 142.

$\ddagger$ Snyder and Sharpe, Certain quartic surfaces bulonging to infinite discontinuous cremonian groups, these TRANSACTIONS, vol. 16 (1915), pp. 62-70. 
$D$ which meets $K_{4}$ in $\left(\gamma_{i}\right)$ also cuts $K_{4}$ in another cubic. Since $C_{4} \sim C_{6}$, the residual $C_{4}-\gamma_{i}$ is transformed into a cubic of genus 1 , hence a plane cubic.

Since $\left[\gamma_{i}, C_{4}\right]=1$, it follows that $\left[\left(\gamma_{i}\right), C_{6}\right]=1$. But $\left[\gamma_{i}, C_{4}-\gamma_{i}\right]=3$, hence $\left[\left(\gamma_{i}\right),\left(C_{4}-\gamma_{i}\right)\right]=3$, and $\left[\left(C_{4}-\gamma_{i}\right)\right]$ meets $\gamma_{i}$ in three points. A plane cubic lying in a plane through $\gamma_{i}$ is therefore transformed into a plane cubic whose plane passes through $\gamma_{i}$. It follows further that $\left[\gamma_{i},\left(\gamma_{i}\right)\right]=0$, but $\left[\gamma_{i},\left(\gamma_{k}\right)\right]=2$.

The surface of trisecants of $C_{6}$ is of order 8 and genus 3 , having $C_{6}$ for triple curve. The intersection of this ruled surface with $K_{4}$ consists of the ten lines $\gamma_{i}$ and a curve of order 4 and genus 3 , hence a plane curve. Thus,

$$
8 C_{4} \equiv 3 C_{6}+\sum \gamma_{i}+C_{4} \quad \text { or } \quad 7 C_{4} \equiv 3 C_{6}+\sum \gamma_{i} .
$$

The 12 systems $\left|C_{4}\right|,\left|C_{6}\right|, \gamma_{i}$ are therefore not independent.

But if we form the determinant of eleven systems, omitting $\gamma_{1}$, it is found to be different from zero. Hence these eleven are independent. It is convenient, however, to retain all 12 systems which satisfy the preceding identity.

21. Transformation of conjugate points on $K_{4}$. When we can express the images of the curves of the base under any transformation, the image of any other system is determined. The transformation $x_{i}^{\prime}=D_{i}$ is that of conjugate points as to the web. It is defined by the equations

S

$$
C_{4} \sim C_{6},
$$$$
C_{6} \sim C_{4} \text {, }
$$$$
\gamma_{i} \sim C_{6}-C_{4}+\gamma_{i} \equiv\left(\gamma_{i}\right) \text {. }
$$

This transformation will be denoted by $S$.

22. Transformation by bisecants of $C_{6}-C_{4}+\gamma_{i}$. The bisecant of the space cubic $\left(\gamma_{i}\right)$ through $P$ on $K_{4}$ meets $K_{4}$ in the residual point $P^{\prime}$. The transformation $P \sim P^{\prime}$ is involutorial. The bisecants from points of a plane section $C_{4}$ describe a ruled surface $R_{10}$ of order ten on which $\left(\gamma_{i}\right)$ is five-fold. The residual intersection with $K_{4}$ is the image of $C_{4}$, apart from the nine lines $\gamma_{k}(k \neq i)$. It has the symbol $10 C_{4}-C_{4}-5\left(C_{6}-C_{4}+\gamma_{i}\right)-\left(\sum \gamma-\gamma_{i}\right)$. Hence

$$
C_{4} \sim 7 C_{4}-2 C_{6}-4 \gamma_{i} .
$$

The bisecants of $\left(\gamma_{i}\right)$ from points on $\gamma_{i}$ describe a ruled surface $R_{4}$ of order 4 , having $\left(\gamma_{i}\right)$ for double curve. Since no other $\gamma_{k}$ intersects $\gamma_{i}, \gamma_{k}$ can not lie on the ruled surface. The complete intersection of $R_{4}$ and $K_{4}$ consists of $\gamma_{i},\left(\gamma_{i}\right)$ counted twice, and a residual curve of order 9 , the proper image of $\gamma_{i}$. Hence

$$
\gamma_{i} \sim 6 C_{4}-2 C_{6}-3 \gamma_{2} .
$$


The lines $\gamma_{k}$ are all bisecants of $\left(\gamma_{i}\right)$, hence remain invariant. The image of $C_{6}$ could be obtained in the same way, but is found more easily from the fact that the identity must remain invariant. We find

$$
C_{6} \sim 12 C_{4}-3 C_{6}-8 \gamma_{i} .
$$

Collecting these results, we have

$\mathrm{T}$

$$
\begin{aligned}
C_{4} & \sim 7 C_{4}-2 C_{6}-4 \gamma_{i}, \\
C_{6} & \sim 12 C_{4}-3 C_{6}-8 \gamma_{i}, \\
\gamma_{i} & \sim 6 C_{4}-2 C_{6}-3 \gamma_{i}, \\
\gamma_{k} & \sim \gamma_{k} .
\end{aligned}
$$

This transformation will be designated by $T$. There are ten different transformations of type $T$.

23. Transformations by transversals of $\gamma_{i}, \gamma_{k}$. This transformation is also involutorial. The transversals which meet $C_{4}$ form an $R_{6}$ having $\gamma_{i}, \gamma_{k}$ for triple lines, hence

$$
C_{4} \sim 5 C_{4}-3 \gamma_{i}-3 \gamma_{k} .
$$

Each curve of $\left|C_{4}-\gamma_{i}\right|,\left|C_{4}-\gamma_{k}\right|$ is invariant, hence

$$
\begin{aligned}
\gamma_{i} & \sim 4 C_{4}-2 \gamma_{i}-3 \gamma_{k}, \\
\gamma_{k} & \sim 4 C_{4}-3 \gamma_{i}-2 \gamma_{k} .
\end{aligned}
$$

The transversals of $\gamma_{i}, \gamma_{k}$ and any other line $\gamma_{n}$ describe a quadric surface, so that

$$
\gamma_{n} \sim 2 C_{4}-\gamma_{i}-\gamma_{k}-\gamma_{n} .
$$

Using the same method as before for $C_{6}$ we obtain

$$
C_{6} \sim 6 C_{4}-C_{6}-3 \gamma_{i}-3 \gamma_{k} .
$$

There are 45 transformations of this type; they will be designated by $U$.

24. Transformations by secants of $\gamma_{i},\left(\gamma_{k}\right)$. This is also involutorial. Using the same methods as before, we find for this transformation

V

$$
\begin{aligned}
C_{4} & \sim 12 C_{4}-3 C_{6}-3 \gamma_{i}-7 \gamma_{k}, \\
C_{6} & \sim 17 C_{4}-4 C_{6}-3 \gamma_{i}-11 \gamma_{k}, \\
\gamma_{i} & \sim 5 C_{4}-C_{6}-2 \gamma_{i}-3 \gamma_{k}, \\
\gamma_{k} & \sim 11 C_{4}-3 C_{6}-3 \gamma_{i}-6 \gamma_{k}, \\
\gamma_{n} & \sim 3 C_{4}-C_{6}-\gamma_{i}-\gamma_{k}-\gamma_{n} .
\end{aligned}
$$

There are 90 of these transformations; they will be designated by $V$. 
The product of any two of the transformations $S, T, U, V$ is non-periodic, hence these operations generate a discontinuous group of infinite order.

25. Transformation of $K_{4}$ into the symmetroid. Under the transformation $x_{1}^{\prime}=A_{1}, x_{2}^{\prime}=B_{1}, x_{3}^{\prime}=C_{1}, x_{4}^{\prime}=D_{1}$, the equation of $K_{4}$ is transformed into $\Delta^{\prime}=0$, in which $\Delta^{\prime}$ is a symmetric determinant, the elements being linear functions of $x^{\prime}$. This is the equation of the symmetroid.*

The fundamental sextic in $(x)$ belongs to the system $C_{6}$. The curves of the other system $\bar{C}_{6}$ are transformed into plane sections $C_{4}^{\prime}$ of the symmetroid. The plane sections $C_{4}$ of $K_{4}$ are transformed into a system of sextics $C_{6}^{\prime}$ on the symmetroid, passing through the ten double points $P_{i}^{\prime}$ images of the lines $\gamma_{i}$. On account of the symmetry, there is only one such system of sextics. They are cut out in pairs by the cubic surfaces through the ten double points $P^{\prime}$, which are the images of the planes in $(x)$.

Hence

$$
2 C_{6}^{\prime}=3 C_{4}^{\prime}-\sum P_{i}^{\prime} ;
$$

this is the transform of the identity in $(x)$. The relations between the two systems are now

$$
C_{4} \sim C_{6}^{\prime}, \quad C_{6} \sim 3 C_{6}^{\prime}-C_{4}^{l}, \quad \gamma_{i} \sim P_{i}^{\prime} .
$$

26. The general quartic surface through a sextic curve of genus 3 . The transformation $x_{i}^{\prime}=D_{i}$ sends $F=\left|a_{1} b_{2} c_{3} d_{4}\right|=0$ into a similar quartic $F^{\prime}=0$, the transformation $x_{1}^{\prime}=A_{1}, x_{2}^{\prime}=B_{1}, x_{3}^{\prime}=C_{1}, x_{4}^{\prime}=D_{1}$ sends $F=0$ into a similar quartic $F^{\prime \prime}=0$. By a transformation of the same kind $F^{\prime}=0$ can be transformed into $F=0$ or into $F^{\prime \prime}=0$, and $F^{\prime \prime}=0$ into $F=0$ or into $F^{\prime}=0$. Hence we may transform $F=0$ into $F^{\prime}=0$, then $F^{\prime}=0$ into $F^{\prime \prime}=0$, and finally $F^{\prime \prime}=0$ into $F=0$. The series of transformations may be expressed as follows:

$$
T_{1} \begin{gathered}
C_{4} \sim C_{6}^{\prime}, \\
C_{6} \sim C_{4}^{\prime},
\end{gathered} \quad T_{2} \begin{gathered}
C_{4}^{\prime} \sim 3 C_{4}^{\prime \prime}-C_{6}^{\prime \prime}, \\
C_{6}^{\prime} \sim 8 C_{4}^{\prime \prime}-3 C_{6}^{\prime \prime},
\end{gathered} \quad T_{3} \quad \begin{gathered}
C_{4}^{\prime \prime} \sim 3 C_{4}-C_{6}, \\
C_{6}^{\prime \prime} \sim C_{4} .
\end{gathered}
$$

Hence the transformation

$$
\begin{array}{rll}
T_{1} T_{2} T_{3} \quad C_{4} \sim 21 C_{4}-8 C_{6}, \\
C_{6} \sim 8 C_{4}-3 C_{6}
\end{array}
$$

leaves $K$ invariant. It is not periodic. $\dagger$

* Cayley, Collected Works, vol. 7, pp. 133-181. See p. 134.

† See Snyder and Sharpe, loc. cit., p. 65. It is readily verified that $T_{1} T_{2} T_{3}$ is identical with $\tau^{3}$. The transformation $\tau^{2}$ is non-existent. That the transformation $F \sim F^{\prime}$, etc., lead to a non-periodic transformation which leaves $F=0$ invariant is mentioned without proof by Cayley, loc. cit., p. 159.

Cornell University December, 1917 